\title{
Argón plasma versus mucosectomía con bandas en el manejo endoscópico del esófago de Barrett con displasia 0 el carcinoma de esófago in situ
}

\section{Argon Plasma Ablation versus Band Mucosectomy for Endoscopic Management of Barrett's Esophagus with dysplasia or Esophageal Carcinoma}

\author{
Rodrigo Castaño, MD, ${ }^{\prime}$ Oscar Álvarez, MD, ${ }^{2}$ Amy Piñeres, MD, ${ }^{3}$ Mario H Ruiz, MD, ${ }^{4}$ Andrés Rojas, MD, ${ }^{5}$ Alejandra Álvarez, ${ }^{6}$ \\ Luis Miguel Ruiz, ${ }^{6}$ David Restrepo, ${ }^{7}$ Víctor Calvo. ${ }^{8}$
}

\author{
Cirugía Gastrointestinal y Endoscopia. Jefe \\ de Postgrado Cirugía General U.P.B, Grupo \\ Gastrohepatología Universidad de Antioquia, Instituto \\ de Cancerología Clínica las Américas. Medellín- \\ Colombia \\ 2 Gastroenterólogo, Texas Valley Coastal Bend \\ (Veterans Administration) y Clinical Assistant \\ Professor UTHSCSA. Estados Unidos \\ ${ }^{3}$ Cirujano General, Clínica Bolivariana. Medellín- \\ Colombia \\ ${ }^{4}$ Cirujano General, Hospital Pablo Tobón Uribe. \\ Medellín-Colombia \\ 5 Cirujano General, Instituto de Cancerología-Clínica \\ las Américas. Medellín-Colombia \\ ${ }^{6}$ Estudiantes de Pregrado, Facultad de Medicina \\ U.P.B. Medellín-Colombia \\ Estudiante de Pregrado, Facultad de Medicina CES. \\ Medellín-Colombia \\ ${ }^{8}$ Estadístico. Medellín-Colombia
}

Fecha recibido: $\quad 31-03-14$ Fecha aceptado: 05-11-14

\begin{abstract}
Resumen
Introducción: se han descrito diferentes técnicas endoscópicas para la ablación del epitelio metaplásico esofágico manteniendo la integridad de la submucosa, bajo una agresiva terapia supresora de ácido con los bloqueadores de bomba. En lo evaluado hasta la fecha existen muy pocos estudios que comparen las diferentes técnicas endoscópicas que reviertan el esófago de Barrett (EB) con displasia y los factores que pueden influir en el pronóstico con estas terapias no se han evaluado consistentemente.

Objetivo: este es un estudio piloto que compara la eficacia en la erradicación completa del EB con displasia, entre la resección endoscópica mucosa (EMR) con bandas y la terapia con argón plasma (APC) con bajo voltaje. Los puntos a evaluar son: a) la completa ablación del EB; b) el número de sesiones requeridas; c) las complicaciones.

Métodos: la APC se realizó con un catéter flexible $10 \mathrm{Fr}$. El voltaje utilizado fue de $50 \mathrm{~W}$ y el flujo varió entre 1 y 2 l/min. En el grupo de EMR con bandas, la mucosa con EB con un equipo de ligadura multibandas. Hasta seis bandas fueron colocadas por sesión. La mucosa con el EB fue removida con un asa de polipectomía con corriente mezclada. Los pacientes fueron seguidos con endoscopia, se obtuvieron biopsias de cuatro cuadrantes en las áreas tratadas o no a los tres, seis y doce meses después de terminar el tratamiento. Todos los pacientes recibían dosis altas de bloqueadores de bomba de protones.

Resultados: este es un estudio prospectivo y describe nuestros resultados en un grupo de 62 pacientes con Barrett y displasia, 33 pacientes (22 hombres, 67\%) tratados con APC y 29 con EMR (19 hombres, 66\%). La ablación completa del EB se dio en $86,2 \%$ con EMR y $79 \%$ en la terapia con APC. El número de sesiones requeridas para lograr el tratamiento completo fue de $1 \pm 1$ con EMR y de $2 \pm 1$ con APC. No se presentaron complicaciones mayores como sangrado digestivo con necesidad de transfusión o perforaciones. La única complicación observada fue la presentación de estenosis de esófago en dos pacientes (una en cada grupo)

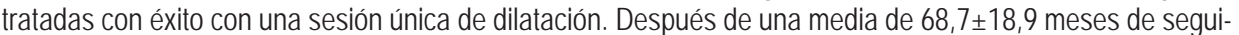
miento en el grupo de APC y de 50,2 $\pm 19,3$ meses en los de EMR, no se observó la presencia de carcinoma de esófago o muertes atribuibles a la enfermedad o la terapia.

Conclusiones: en los pacientes con EB con displasia de alto o bajo grado, el APC o la EMR logran comparables altas tasas de erradicación de la neoplasia y la metaplasia intestinal (79\% versus $86 \%$ ). Ambos procedimientos son equiparables en efectividad y bastantes seguros con una baja tasa de complicaciones; sin embargo, APC se asocia a un mayor número de sesiones.
\end{abstract}

Palabras clave

Esófago de Barrett, reflujo gastroesofágico, argón plasma, displasia en esófago. 


\begin{abstract}
Introduction: Various endoscopic techniques for ablation of metaplastic esophageal epithelia which maintain the integrity of the submucosa have been described. All maintain patients under aggressive acid suppressive therapy with proton pump inhibitors. To date, few studies have compared the different endoscopic techniques for eradicating Barrett's esophagus (BE) with dysplasia. Similarly the factors that might influence patients' prognoses after these therapies have not been very consistently evaluated.

Objective: This is a pilot study which compares the effectiveness of complete eradication of BE with dysplasia through endoscopic mucosal resection (EMR) with bands and low voltage endoscopic argon plasma coagulation (APC). The three points evaluated are whether complete ablation of BE was achieved, the number of sessions required and whether and what complications occurred.

Methods: APC was performed using a flexible 10 French catheter. The voltage used was $50 \mathrm{~W}$ and flow timed varied between 1 minute and $2 \mathrm{l} / \mathrm{min}$. In one group BE mucosa was treated with multi-band ligation. Up to six bands were placed per session. The mucosa with BE was removed with a polypectomy loop and a mixed stream of water. Patients were followed up endoscopically and biopsies were obtained from the four quadrants in both treated and untreated areas at three, six and twelve months after treatment ended. All patients received high doses of proton pump inhibitors.

Results: This prospective study describes our results in a group of 62 patients with Barrett's Esophagus and dysplasia. Thirty-three patients (22 men, 67\%) were treated with APC and 29 were treated with EMR (19 men, $66 \%$ ). Complete ablation of BE was achieved in $86.2 \%$ of patients treated with EMR and in $79 \%$ of those treated with APC. The number of sessions required to achieve complete ablation was $1 \pm 1$ for EMR and 2 \pm 1 for APC. No major complications such as gastrointestinal bleeding requiring transfusion or perforations occurred. The only complications were two cases of esophageal stenosis, one in each group. These were successfully treated with single sessions of dilation. After a mean of $68.7 \pm 18.9$ months of follow-up in the APC group and $50.2 \pm 19.3$ months in the EMR group no cases of carcinoma of the esophagus and no deaths due to disease or therapy had occurred.
\end{abstract}

Conclusions: In patients with BE with high or low grade dysplasia, APC and EMR achieve comparably high eradication rates of neoplasia and intestinal metaplasia ( $79 \%$ versus $86 \%$ ). Both procedures have comparable levels of effectiveness and safety and have low rates of complications. Nevertheless, APC is associated with a greater number of sessions.

\title{
Keywords
}

Barrett's esophagus, gastroesophageal reflux, argon plasma, dysplasia in the esophagus.

\section{INTRODUCCIÓN}

El esófago de Barrett (EB) es una lesión precancerosa adquirida, donde el epitelio escamoso del esófago distal es reemplazado por células columnares en las cuales se demuestran características de la metaplasia intestinal (1). El riesgo de desarrollar carcinoma se aumenta al incrementarse el grado de displasia (2). El adenocarcinoma de esófago (CE) tiene un pobre pronóstico a pesar del tratamiento y a su reconocido incremento en la incidencia en todo el mundo, mayor a cualquier otra neoplasia $(3,4)$. A pesar de reconocer el reflujo gastroesofágico crónico como el factor desencadenante de la metaplasia intestinal, el control del reflujo por terapia médica (5) o quirúrgica (6) no ha mostrado un impacto favorable reversando la metaplasia intestinal. Hasta el presente, no es posible reconocer las alteraciones genéticas celulares que marcarán los cambios del EB hacia la displasia o el cáncer (7), por lo que algunos proponen un seguimiento endoscópico para detectar tempranamente los cambios displásicos $(8,9)$.
La esofagectomía ha sido recomendada tradicionalmente para los pacientes con displasia de alto grado (DAG) y/o cáncer de esófago y se reconoce que no es un procedimiento exento de grandes riesgos de morbimortalidad, especialmente en pacientes ancianos y/o con comorbilidades (10), por lo tanto, la terapia endoscópica se constituye en una alternativa adecuada para estos pacientes (11). Dos de estos métodos de manejo endoscópico, de fácil acceso en nuestro medio, son la ablación con argón plasma (APC) y la resección endoscópica mucosa (MER) con bandas (12). Estas terapias, en los casos de EB con DAG y el carcinoma intramucoso, están justificadas por la ausencia de compromiso ganglionar locorregional.

Las terapias ablativas locales o limitadas a la displasia presentan una recaída en el seguimiento hasta de 30\% (13-15). Por esto, la meta de la terapia ablativa para el EB es la completa erradicación del epitelio metaplásico y el reemplazo de este por un epitelio neoescamoso sin el potencial de malignidad que tiene la metaplasia intestinal especializada, con una tasa baja de complicaciones (16-19). No hay evi- 
dencia que sustente la necesidad de la erradicación del EB sin displasia. Recientemente en nuestro medio, se describió la técnica de la terapia con APC y se demostró en un estudio con seguimiento prospectivo que la terapia con APC en el EB displásico erradica $69 \%$ de este epitelio, en un seguimiento cercano a los cuatro años en promedio (20). Una gran limitante de las terapias ablativas es la ausencia de un espécimen histológico, lo que difiere la valoración del éxito o fracaso de la terapia.

Con la EMR sola o combinada con terapias ablativas se obtiene un material histológico, particularmente importante en el EB con nodulaciones, en los casos de carcinoma intramucoso provee información respecto a la diferenciación de este, presencia o ausencia de infiltración linfovascular, profundidad de la invasión y si hay compromiso o no de los márgenes de resección (21). No consideramos una buena práctica la técnica endoscópica que describe la ligadura sin resección de la mucosa (22).

Técnicas de EMR descritas incluyen la resección con asa de polipectomía, levantar con inyección submucosa y cortar, resecciones asistidas por capuchones, resecciones con ligaduras (11). La ligadura y posterior resección es un método simple y fácil de implementar y es extrapolado a partir de la experiencia con la ligadura de las várices esofágicas $(23,24)$. La resección con bandas se puede hacer con o sin la inyección previa de cualquier solución, aspirando el tejido a un capuchón para luego liberar la banda; esto crea un pseudopólipo que luego es resecado con el asa de polipectomía y recuperado para su estudio histológico. La resección asistida por capuchón ha sido comparada con la resección con bandas, con igual seguridad y eficacia (25).

En el presente estudio, se pretende comparar la eficacia para lograr la erradicación completa del EB con displasia o carcinoma in situ entre el APC y la EMR en diferentes centros de tercer nivel en la ciudad de Medellín-Colombia.

\section{MATERIALES Y MÉTODOS}

Los pacientes fueron remitidos para el manejo de EB con displasia de alto o bajo grado en el periodo comprendido entre junio de 2004 y noviembre de 2011, en dos centros hospitalarios de tercer nivel de la ciudad de Medellín, Colombia.

Todos los pacientes tenían, en los dos meses previos al procedimiento, una endoscopia con un protocolo de biopsias de cada $2 \mathrm{~cm}$ en los cuatro cuadrantes. A los pacientes con hallazgo por las biopsias de cáncer invasor más allá de la mucosa (T1 sm o más profundo) se les recomendó la resección quirúrgica. A ningún paciente se le realizó estudio con ultrasonido endoscópico.

Los pacientes inicialmente (desde 2004) fueron manejados con terapia con APC hasta el 2009. A partir de enero de 2006, se introdujo la terapia de mucosectomía con bandas, no necesariamente se utilizó el kit Duette de mucosectomía multibandas (6 casos); en la mayoría de pacientes se realizó la ligadura con el kit de bandas para várices y la resección del tejido ligado se hizo con un asa de polipectomía de 10 o $20 \mathrm{~mm}$ (figura 1).

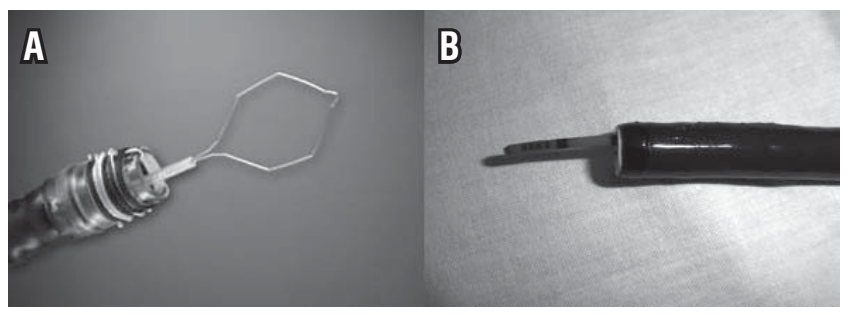

Figura 1. A. Kit Duette de mucosectomía con bandas (Six-Shooter, Wilson-Cook Inc, Winston-Salem, NC) montado en el endoscopio con el asa de polipectomía a través del canal de trabajo. B. Endoscopio con catéter en el canal de trabajo, para la aplicación del argón plasma con una potencia preajustada de 60 Watts y con un flujo del gas de $11 / \mathrm{min}$.

Se excluyeron pacientes con neoplasias en otros sitios, resecciones en esófago, cáncer avanzado de esófago, pacientes embarazadas, pacientes mayores de 80 años o aquellos con enfermedades terminales.

El protocolo y aprobación del estudio se hizo por el comité de ética de la Universidad de Antioquia que evalúa los protocolos de investigación de los residentes.

\section{Evaluación antes del procedimiento}

Se obtuvo el consentimiento informado para todos los pacientes. La longitud del EB se definió como la distancia entre el margen de los pliegues gástricos proximales y la unión escamo-columnar. El mapeo endoscópico del EB se hizo en los cuatro cuadrantes cada dos $\mathrm{cm}$ con una pinza de biopsia estándar, o bien las biopsias se dirigieron específicamente a las lesiones cuando eran visibles (cambios de coloración, nódulos, ulceraciones, etc.)

\section{Técnicas de terapia endoscópica}

\section{Mucosectomía con bandas}

Los pacientes fueron sedados con una combinación de midazolam y meperidina intravenosas, no se utilizó propofol en ninguno y no hubo efectos adversos atribuibles a la sedación. El kit Duette de mucosectomía con bandas (Wilson Cook Medical- Bloomington, IN) incluye un ligador multibandas y un asa de polipectomía hexagonal de 1,5 por $2,5 \mathrm{~cm}$ hecha de alambre trenzado (figura 1A). En la mayoría de pacientes se utilizó el original ligador multibandas de Saeed (WilsonCook Medical, Winston-Salem, NC). Una vez el ligador es montado en la punta del endoscopio, se avanza hasta el esófago y se realiza la aspiración y ligadura de la mucosa en 
forma de pseudopólipo, hasta por 6 bandas con el kit Duette y hasta 10 con el kit de Saeed (figura 2).

La energía de corte fue de 30 Watt con corriente mixta. No se realizó ninguna inyección de la submucosa antes de la succión y ligadura. La siguiente aplicación se hace en la mucosa inmediatamente adyacente al margen de resección. Se registraron el número de terapias, longitud del EB displásico tratado y las reacciones adversas inmediatas o tardías. La extensión de la ligadura se individualizó de acuerdo a la longitud y configuración del EB y/o las lesiones macroscópicas, la presencia de uni o multifocalidad de la displasia. El producto de la resección se llevó al estómago y allí se recogieron o con un canastillo de Dormia o con una malla atrapa-pólipos (Roth Net) y luego fueron fijados en formol al 10\% para estudio histopatológico. Los pacientes se dieron de alta el mismo día luego de recuperarse de la sedación, con instrucciones de doble dosis de bloqueador de bomba (por 10 días), sumado a cuatro dosis diaria de 15 $\mathrm{ml}$ de sucralfato (por 10 días), más acetaminofén $(500 \mathrm{mg}$ ) con codeína $(8 \mathrm{mg})$ por una semana y una dieta licuada por tres días y después de esto una dieta normal.

\section{Terapia con argón plasma}

Diseñado inicialmente para la cirugía abierta, es un procedimiento de electrocoagulación sin contacto para uso endoscópico. El gas argón es provisto a través de un catéter de teflón insertado a través del canal de trabajo del endoscopio y el argón es ionizado con una unidad electroquirúrgica con corriente de alta frecuencia (figura 1B). Se adquiere una temperatura de hasta $130{ }^{\circ} \mathrm{C}$ que lleva a la desecación del tejido con un máximo de profundidad de 3 a $4 \mathrm{~mm}$, ideal para la ablación del epitelio metaplásico en el EB. Bajo sedación con midazolam más meperidina y por el canal del trabajo del endoscopio el gas argón se administra en forma rectilínea por el catéter o bien en forma radial o lateral al encontrar el tejido conductivo. La APC se aplicó con una potencia de 60 Watts y a un flujo de $11 /$ min y se iniciaba desde la unión GE y se progresaba en sentido proximal. De acuerdo con los principios físicos el gas argón tiene la tendencia a ir de áreas coaguladas (alta impedancia) a las inadecuadamente coaguladas (baja impedancia) (figura 3).

La principal ventaja de APC es su fácil movilidad, bajo costo, no tiene los riesgos biológicos del láser ni los requerimientos de licencia para su uso. El número de sesiones dependió de la extensión del EB y de la tolerabilidad del paciente a la terapia.

Después de la intervención los pacientes se dieron de alta el mismo día luego de recuperarse de la sedación, con instrucciones de doble dosis de bloqueador de bomba (por 10 días), más acetaminofén $(500 \mathrm{mg})$ con codeína $(8 \mathrm{mg})$
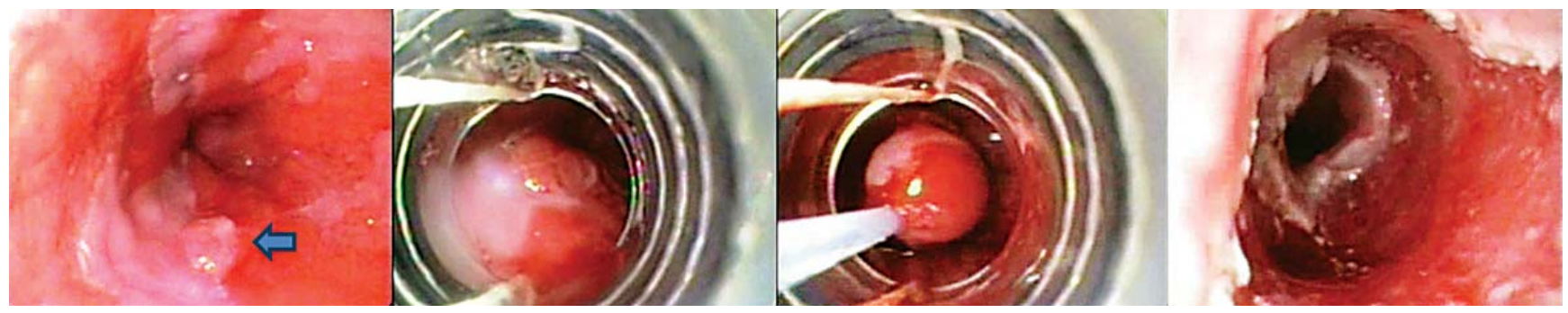

Figura 2. Secuencia de la terapia con bandas a EB con displasia. A. Nódulo con displasia de alto grado en EB extenso. B. Bandas montadas en el endoscopio y nódulo distal. C. Captura con asa de mucosa ligada con banda. D. Aspecto del lecho tras la resección con asa de la mucosa.

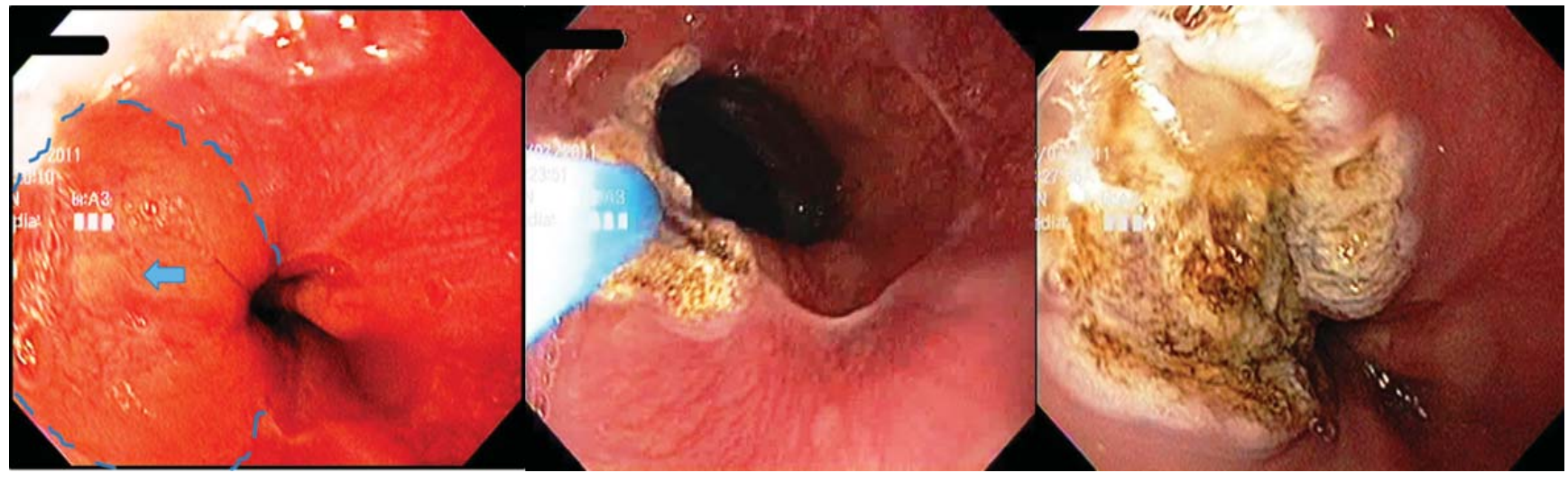

Figura 3. Aspectos endoscópicos de la terapia con argón plasma. A. Lengüeta de Barrett corto destacada por guiones con nodulación central (flecha) con displasia de bajo grado. B. Inicio de la terapia con argón plasma a 60 Watts con 1 l/min de flujo que va de distal a proximal. C. Terapia con argón plasma terminada donde se logró la ablación de la lengüeta del Barrett y su nódulo central. 
por una semana, con dieta licuada por tres días y después de esto una dieta normal. Los pacientes fueron contactados telefónicamente entre 5 y 7 días después de la intervención para evaluar los efectos adversos de la terapia.

\section{Patología}

La evaluación histológica incluye la descripción de lo adecuado del espécimen para la interpretación histológica, presencia del epitelio especializado y el grado de displasia. La displasia se definió como el epitelio neoplásico confinado hasta la membrana basal en ausencia de inflamación y la gradación de acuerdo con la clasificación de Viena $(26,27)$ en negativo para displasia, indefinido para displasia, DBG, $\mathrm{DAB}$ y carcinoma. Cuando el cáncer estaba presente se determinó la profundidad de invasión, grado de diferenciación (bien o mal diferenciado) invasión linfática, vascular o neural, y si hay o no compromiso de los márgenes de resección, laterales y en profundidad. Los márgenes laterales no pueden ser evaluados correctamente en los casos de resecciones en fragmentos. Un cáncer mucoso T1A se definió como una lesión con una invasión en profundidad limitada a la muscularis mucosa y la variedad T1B si la extensión iba hasta la submucosa sin comprometer la muscular propia.

Con las biopsias durante el seguimiento endoscópico del nuevo epitelio escamoso se evaluó la presencia de metaplasia intestinal en el epitelio o por debajo de este (metaplasia intestinal subescamosa o Barrett oculto o sumergido).

\section{Seguimiento}

Se definió la erradicación completa de la MI por histología y endoscopia, si tras 2 endoscopias consecutivas con biopsia (en esófago distal y unión gastroesofágica) de cuatro cuadrantes cada $1 \mathrm{~cm}$ con pinza de biopsia estándar no se documentaba la presencia de metaplasia intestinal. La recurrencia se definió como la presencia de histológica de metaplasia intestinal con o sin displasia después de adquirir la erradicación completa del EB displásico.

Los pacientes recibían durante el seguimiento $40 \mathrm{mg}$ de un bloqueador de bomba de protones (omeprazol o esomeprazol) y fueron tratados endoscópicamente cada 6-12 semanas hasta lograr la erradicación endoscópica del EB. El seguimiento endoscópico postratamiento se hace a los $3,6,12,18$ y 24 meses y luego cada año, con biopsias de cuatro cuadrantes del epitelio "neoescamoso" en el área intervenida.

\section{Análisis estadístico}

En el análisis descriptivo se utilizaron distribuciones absolutas, relativas e indicadores de resumen como la media arit- mética, la desviación estándar, la mediana y el rango intercuartílico. Para la relación de aspectos personales y clínicos según tipo de técnica se utilizó la prueba Chi cuadrado de independencia o la prueba Exacta de Fisher o la prueba de Likelihood Ratio cuando fue necesario. Se estableció el criterio de normalidad mediante la prueba de ShapiroWilk y con base en esta se aplicó la prueba t-Student para la diferencia de medias independientes o la prueba $U$ de Mann-Whitney. Se realizó un análisis de supervivencia por el método de Kaplan-Meier y las curvas obtenidas se compararon mediante la prueba Log Rank (Mantel-Cox). Un valor p inferior de 0,05 se consideró estadísticamente significativo. La sistematización, el procesamiento y análisis de los datos se realizó mediante el programa SPSS versión 21.

\section{RESULTADOS}

En un periodo de 7 años, a 62 pacientes (41 hombres, 66\%) se les practicaron terapias para la erradicación con ablación o resección del EB displásico; 33 pacientes fueron tratados con APC y a 29 se les realizó mucosectomía con bandas. La edad osciló entre los 48 y 72 años ( $63 \pm 4,8$ años).

En ambos grupos se evaluaron los años con manifestaciones de reflujo gastroesofágico, antecedente de cirugía antirreflujo, consumo de cigarrillos, presencia de hernia hiatal, el índice de masa corporal, y la presencia o no de nódulos asociados al EB (tabla 1).

Respecto a los hallazgos endoscópicos y otros aspectos clínicos, se evaluó además del grado de displasia, la presencia o no de nódulos en el EB, la longitud promedio del Barrett circunferencial (BC) y el margen más alto del Barrett (BM), número de sesiones, presencia de complicaciones y el tiempo promedio de terapia. En el grupo mucosectomía con bandas había 8 pacientes con terapias previas con APC con la intención de remover la displasia en el Barrett (tabla 2).

Los pacientes en ambos grupos tuvieron dolor después de las intervenciones con APC (24\%) y las bandas (24\%). Solo dos pacientes (una en cada grupo) ameritó dilatación por estenosis. Un paciente en el grupo con APC requirió hospitalización por 48 horas para manejo de dolor torácico, odinofagia y fiebre, manejada con analgésicos y líquidos intravenosos, con recuperación completa. No hubo perforaciones y pocos pacientes presentaron sangrado evidenciado en dos casos durante la terapia con APC y uno en la terapia con bandas, pero no hubo necesidad de transfusiones en ninguno.

Los resultados del examen histopatológico tras la resección endoscópica mucosa con bandas mostró cambios en 11 pacientes, en 8 hubo un incremento en el hallazgo histológico (6 de displasia de bajo a alto grado 2 de DAG a carcinoma, uno in situ y otro con invasión a la submucosa), 
Tabla 1. Distribución de aspectos demográficos y clínicos según tipo de técnica.

\begin{tabular}{|c|c|c|c|c|}
\hline & \multicolumn{2}{|c|}{ Técnica } & \multirow[t]{2}{*}{ Valor $p$} \\
\hline & & APC (\%) & Bandas (\%) & \\
\hline \multirow[t]{2}{*}{ Sexo } & Hombre & $22(66,7)$ & $19(65,5)$ & 0,924 \\
\hline & Mujer & $11(33,3)$ & $10(34,5)$ & \\
\hline \multirow[t]{2}{*}{ Fuma } & $\mathrm{Si}$ & $15(45,5)$ & $18(62,1)$ & 0,191 \\
\hline & No & $18(54,5)$ & $11(37,9)$ & \\
\hline \multirow[t]{2}{*}{ Anti-RGE } & Si & $9(27,3)$ & $7(24,1)$ & 0,778 \\
\hline & No & $24(72,7)$ & $22(75,9)$ & \\
\hline \multirow{2}{*}{$\begin{array}{l}\text { Tratamiento } \\
\text { previo }\end{array}$} & Si & $0(0,0)$ & $8(27,6)$ & N.A \\
\hline & No & $33(100)$ & $21(72,4)$ & \\
\hline \multirow[t]{2}{*}{ Hernia hiatal } & $\mathrm{Si}$ & $19(57,6)$ & $18(62,1)$ & 0,719 \\
\hline & No & $14(42,4)$ & $11(37,9)$ & \\
\hline \multirow[t]{2}{*}{ Nódulos } & $\mathrm{Si}$ & $8(24,2)$ & $8(27,6)$ & 0,764 \\
\hline & No & $25(75,8)$ & $21(72,4)$ & \\
\hline \multirow[t]{3}{*}{ Sesiones } & Una sesión & $3(9,1)$ & $19(65,5)$ & 0,000 \\
\hline & Dos sesiones & $17(51,5)$ & $10(34,5)$ & \\
\hline & Tres sesiones & $13(39,4)$ & $0(0,0)$ & \\
\hline \multirow[t]{2}{*}{ Displasia } & Severa & $8(24,2)$ & $9(31,0)$ & 0,550 \\
\hline & Leve & $25(75,8)$ & $20(69,0)$ & \\
\hline \multirow[t]{2}{*}{ Complicaciones } & $\mathrm{Si}$ & $11(33,3)$ & $9(31,0)$ & 0,847 \\
\hline & No & $22(66,7)$ & $20(69,0)$ & \\
\hline \multirow{3}{*}{$\begin{array}{l}\text { ¿Cuál } \\
\text { complicación? }\end{array}$} & Dolor & $8(72,7)$ & $7(77,8)$ & $0,902^{\star *}$ \\
\hline & Sangrado & $2(18,2)$ & $1(11,1)$ & \\
\hline & Estenosis & $1(9,1)$ & $1(11,1)$ & \\
\hline \multirow[t]{2}{*}{ Cirugía } & $\mathrm{Si}$ & $7(21,2)$ & $6(20,7)$ & 0,960 \\
\hline & No & $26(78,8)$ & $23(79,3)$ & \\
\hline \multirow[t]{2}{*}{ Nuevo Barrett } & Si & $7(21,2)$ & $4(13,8)$ & 0,445 \\
\hline & No & $26(78,8)$ & $25(86,2)$ & \\
\hline \multirow[t]{2}{*}{ Nueva displasia } & Si & $4(12,1)$ & $2(6,9)$ & $0,676^{*}$ \\
\hline & No & $29(87,9)$ & $27(93,1)$ & \\
\hline \multirow[t]{2}{*}{ Cáncer } & $\mathrm{Si}$ & $0(0,0)$ & $0(0,0)$ & N.A \\
\hline & No & 33 (100) & $29(100)$ & \\
\hline
\end{tabular}

*Prueba Exacta de Fisher's

**Prueba de Likelihood Ratio.

Tabla 2. Distribución de aspectos personales y clínicos según técnica.

\begin{tabular}{lccc}
\hline & \multicolumn{2}{c}{ Técnica } & Valor $\mathbf{p}$ \\
\cline { 2 - 3 } & APC & Bandas & \\
\hline Edad en años* $^{*}$ & $62,4 \pm 5$ & $63,8 \pm 4,5$ & 0,258 \\
Años con RGE* & $10,4 \pm 1,6$ & $11,1 \pm 3,3$ & 0,319 \\
Paq. cigarrillos/año* & $14(4)$ & $10(5)$ & 0,040 \\
IMC $^{\star *}$ & $28(6)$ & $29(2)$ & 0,642 \\
\# Sesiones & $2(1)$ & $1(1)$ & 0,000 \\
Barrett circular cm $^{\star \star}$ & $3(2)$ & $3(1)$ & 0,592 \\
Barrett margen cm $^{\star \star}$ & $3(1)$ & $4(2)$ & 0,048 \\
Tiempo tratamiento días* & $79(60)$ & $1(64)$ & 0,000 \\
\hline
\end{tabular}

* Los valores expresan: media aritmética \pm desviación estándar

** Los valores expresan: mediana (rango intercuartil). en 3 se bajó el grado de displasia (de DAG a DBG) y en 18 no hubo cambios.

Durante el seguimiento no se presentaron más recaídas del EB (islotes submucosos) o aparición de displasia que se relacionara con la extensión circunferencial o la longitud del margen proximal del EB.

Los dos grupos fueron comparados con base en el protocolo de seguimiento a los 3, 6, 12 y 18 meses. Durante el seguimiento se documentó la recaída del EB en 7 pacientes en el grupo con APC (21\%) y en 4 pacientes en el grupo tratado con mucosectomía con bandas $(13,8 \%)$, la recaída más temprana fue del EB a los 8 meses. La displasia se hizo aparente nuevamente en 4 pacientes tratados con APC y en 2 con mucosectomía, dos de ellos precedidos por recaída del EB. No se presentó ningún caso de cáncer durante el seguimiento (tabla 3 ).

Tabla 3. Hallazgos tras el seguimiento de los dos grupos.

\begin{tabular}{lccc}
\hline & \multicolumn{2}{c}{ Técnica } & \multirow{2}{*}{ Valor $\mathbf{p}$} \\
\cline { 2 - 3 } & APC & Bandas & \\
\hline Nuevo Barrett & 7 & 4 & 0,258 \\
Nueva displasia leve & 4 & 2 & 0,319 \\
Cáncer & 0 & 0 & 0,9 \\
Cirugía anti-RGE & 7 & 6 & 0,642 \\
Seguimiento en meses* & $68,7 \pm 18,9$ & $50,2 \pm 19,3$ & 0,000 \\
\hline
\end{tabular}

*Los valores expresan: media aritmética \pm desviación estándar.

En los pacientes que apareció el EB después de cualquiera de las terapias fueron considerados como fallas en el tratamiento. En tres pacientes se encontró un reporte único de la presencia de EB en las biopsias del seguimiento y esto no se repitió en ninguna de las otras biopsias, 2 pacientes tratados con APC (uno con Barrett sumergido y otro con Barrett epitelial) y otro tratado con REM con bandas (EB epitelial). Estos pacientes no se consideraron fallos de la terapia.

\section{DISCUSIÓN}

La esofagectomía ha sido la terapia estándar para el manejo de los pacientes con displasia de alto grado o carcinoma intramucoso y aunque se logra la curación, la morbimortalidad que la intervención implica es muy alta, especialmente en centros sin mucho volumen quirúrgico con este tipo de pacientes (28).

El objetivo de la terapia endoscópica ablativa en el esófago de Barrett con displasia es lograr la erradicación completa de la metaplasia intestinal y la displasia, manteniéndose a largo plazo y con una mínima posibilidad de complicaciones. 
En este estudio se compara el APC y la resección endoscópica mucosa con bandas del EB con displasia. Con el incremento del seguimiento endoscópico y los protocolos de biopsia aplicados en el EB, se espera que cada vez se detecten más casos de displasia. Tanto para la displasia como para el cáncer de esófago intramucoso, la terapia endoscópica representa una beneficiosa realidad. La terapia endoscópica temprana con técnicas ablativas como el APC, terapia fotodinámica, radiofrecuencia o la resección mucosa llevan a un mejor pronóstico. La intención de estas terapias es proveer una sobrevida similar a la obtenida por la cirugía, con menos morbilidad, menor mortalidad y con una mejor relación costo-beneficio. La técnica de ablación ideal o la combinación de estas no se ha identificado aún. El propósito de este estudio es comparar dos técnicas de ablación como la APC y la mucosectomía endoscópica con bandas con un seguimiento prospectivo en pacientes con EB con displasia.

En 1998 se publicaron los primeros estudios de terapia con APC en EB por Mork (29) y Byrne (30), con unos resultados inicialmente auspiciosos que no se han replicado en estudios más recientes, incluso en nuestro medio (20). No hay consenso respecto a la potencia en Watts ofrecida en la terapia con APC y si bien los mejores resultados como los de Manner (31) (erradicó el 77\% del EB sin displasia) se lograron con 90 Watts de potencia con morbilidad alta y aún no hay evidencia de que la ablación del Barrett sin displasia sea de utilidad. En el presente estudio se utilizó una potencia intermedia de 60 Watts, aunque hay descritas terapias desde 30 Watts (32).

La resección mucosa endoscópica asistida por un capuchón en la punta del endoscopio fue descrita inicialmente por Inoue (33) y esta técnica fue modificada por Fleischer (23) con la aplicación de una banda al tejido esofágico luego de crear un pseudopólipo por aspiración (con o sin inyección de la submucosa), seccionándolo con un asa de polipectomía.

Las terapias con APC han sido comparadas en forma aleatoria con la terapia fotodinámica por Hage (34), Ragunath (35), y Kelty (36) con resultados similares para ambas terapias para el EB con o sin displasia y con unos escasos periodos de seguimiento ( 12 meses), con una relación costo-efectividad que favorece la terapia con APC.

La penetración de la terapia en espesor de la pared el esófago es un determinante fundamental en el éxito de la misma. Ackroyd (37) ha evaluado el espesor del epitelio del esófago con o sin metaplasia intestinal en los bloques de parafina y sugiere que un espesor probable de EB es de 0,6 $\mathrm{mm}$ (ligeramente mayor al epitelio escamoso). El espesor de la ablación con APC y la terapia fotodinámica es más que suficiente para erradicar el tejido displásico y metaplásico de la mucosa esofágica.
La aparición del epitelio metaplásico debajo del epitelio escamoso (EB oculto) fue descrito inicialmente por Barr (38) y se ha encontrado en todos los tipos de terapia ablativa y ya se ha informado la aparición de cáncer en este epitelio $(35,39-42)$.

Los efectos adversos de ambas terapias fueron similares y no se presentaron complicaciones mayores como la perforación. Los casos que ameritaron dilatación en ambos grupos ( $1 \mathrm{de} \mathrm{c} / \mathrm{u})$, resolvió el problema de la estenosis.

Recientemente, modalidades combinadas que involucran la resección mucosa sumadas a terapias ablativas han mostrado mejores resultados. Sin embargo, para tener unas conclusiones fehacientes se sugiere un estudio con 120 pacientes con EB y displasia en cada grupo, para detectar diferencias significativas, seguido por un suficiente tiempo de seguimiento.

No se consideró en el presente estudio la evaluación previa al procedimiento con ultrasonido endoscópico, porque las publicaciones en este sentido indican que hay una sobrestimación de la profundidad del compromiso (43) e incluso en el Barrett nodular lo aconsejable es la remoción del nódulo (44).

El uso del APC para la ablación del EB se ha informado en diferentes estudios $(29,45-51)$ donde la reversión del Barrett se describe entre $42 \%$ y $98 \%$, después de 1 a 6 tratamientos. En el presente estudio la ablación completa del EB con displasia se logró con la terapia con APC en $67 \%$ mientras con la mucosectomía se logró en $79 \%$. Estos resultados no son alentadores y en buena medida se explica por la estricta definición de Barrett persistente aplicada en este estudio; se consideró que cualquier epitelio columnar especializado era un Barrett persistente. Esta situación se hizo aparente en la aparición de islotes subepiteliales durante el seguimiento, con el potencial carcinogénico descrito.

Se ha sugerido que los resultados de la terapia con APC se pueden mejorar aumentando la potencia a más de $65 \mathrm{~W}$, incrementando la profundidad de la terapia con una más completa ablación $(46,47)$. En nuestro estudio se replicó el hallazgo previo que muestra que la recurrencia es más frecuente en los EB de más de $3 \mathrm{~cm}$, con la persistencia del reflujo ácido y en diferentes estudios la terapia con BBP se modula con base en los hallazgos de $\mathrm{pH}$-impedanciometría. Como en este estudio no se realizó el monitoreo del $\mathrm{pH}$, no podemos excluir el hecho que la reaparición del EB se relacione con una mayor exposición a $\mathrm{pH}$ menores de 4 en los pacientes con EB subepitelial.

Hasta hace unos años, el objetivo de la terapia ablativa era controlar la displasia. Sin embargo, estudios recientes han mostrado que las anomalías genéticas pueden persistir después de la terapia ablativa $(52,53)$. Van Hillegersberg (54) describió dos pacientes con cáncer en EB incompletamente abolidos con la terapia ablativa, de allí que el objetivo de la 
terapia sea la desaparición completa del epitelio metaplásico. La presencia de EB después de la primera terapia con APC indica la necesidad de una nueva terapia, hasta lograr erradicar el EB totalmente. Sin embargo, ninguna de las terapias logró la erradicación completa del EB en todos los pacientes.

La calidad del espécimen resecado es suficientemente satisfactoria para determinar la profundidad de la neoplasia en los casos en que se encuentre. Nuestro estudio demuestra cambios en el diagnóstico histológico con dos pacientes con DAG cuyo hallazgo final por la mucosectomía determinó un cáncer intramucoso (en seguimiento) y otro con invasión a la submucosa manejado con esofagectomía sin lesión residual.

La mucosectomía endoscópica con bandas es un procedimiento seguro y efectivo y puede hacer parte de la evaluación, estadificación y erradicación endoscópica de los pacientes con DAG o carcinoma intramucoso. Puede ser aplicada sin la necesidad de una inyección submucosa previa y en pacientes ambulatorios, con sedación y analgesia, sin necesidad de interrumpir la ingesta oral del paciente. Amplias áreas de mucosa pueden ser resecadas en forma rápida con una mayor precisión histológica para un más preciso diagnóstico y estadificación de la lesión. Las estenosis secundarias a la mucosectomía se suceden con resecciones mucosas que comprometan más de $50 \%$ de la circunferencia y en general responden fácil a las dilataciones. El sangrado no es frecuente y se sucede en horas tempranas luego de la intervención y se puede evitar si el corte se hace con coagulación pura (21).

Más recientemente se ha sugerido una terapia combinada para el EB con displasia, iniciando con la REM con bandas y al EB remanente se trata con APC, con menor recurrencia de la displasia durante un seguimiento de dos años (55), combinación terapéutica que fue refrendada en el editorial de la publicación (576).

En nuestro estudio, la erradicación del EB con displasia se logró en todos los pacientes con la terapia inicial, sin embargo, con el seguimiento a largo plazo se hace aparente que la posibilidad de recaída es frecuente a pesar de la ablación inicial, lo que se ha sugerido en estudios recientes (57).

Existen escasas publicaciones que avalen el aspecto de costo efectividad de las terapias ablativas en el EB con displasia; cuando se compara la APC con la terapia fotodinámica las cifras favorecen en términos de costo a la terapia con APC (35), se reconoce que las terapias ablativas para el EB muestran ser costo-efectivas si el tratamiento es definitivo y no se requiere de un seguimiento en el futuro, lo que a la fecha no se puede ofrecer con ninguna de estas terapias.

\section{CONCLUSIONES}

La terapia endoscópica por ablación como APC o por resección con bandas para el EB con displasia ha venido ganando espacio, permitiendo el control de la entidad con una menor morbimortalidad de los pacientes comparada con las intervenciones quirúrgicas más invasivas. El presente estudio, como en la literatura médica en general, no logra identificar cuál es la mejor de estas terapias endoscópicas, pero sí es claro que debe ser temprana y con un número bajo de retratamientos para obtener la erradicación completa.

En nuestro medio, este tipo de terapias pueden ser costoefectivas, aun con seguimientos endoscópicos a largo plazo, debido a los bajos costos de los procedimientos.

Se necesitan estudios con más pacientes y más tiempo de seguimiento para poder establecer cuál es la mejor de las terapias (ablativas o resectivas), si es necesaria la combinación de estas o si definitivamente alcanzan igual rango de curación que la esofagectomía, en términos de periodo libre de enfermedad y calidad de vida.

\section{Conflictos de intereses}

Los autores no presentan ningún conflicto de interés.

Este estudio se realizó con el apoyo del Proyecto sostenibilidad de la vicerrectoría de investigación de la Universidad de Antioquia.

\section{REFERENCIAS}

1. Leeuwenburgh I, Scholten P, Calje TJ, et al. Barrett's esophagus and esophageal adenocarcinoma are common after treatment for achalasia. Dig Dis Sci 2013; 58: 244-52.

2. Hur C, Miller M, Kong CY, et al. Trends in esophageal adenocarcinoma incidence and mortality. Cancer 2013; 119: 1149-58.

3. Thrift AP, Kendall BJ, Pandeya N, Whiteman DC. A model to determine absolute risk for esophageal adenocarcinoma. Clin Gastroenterol Hepatol 2013; 11: 138-44 e2.

4. Pohl H, Wrobel K, Bojarski C, et al. Risk factors in the development of esophageal adenocarcinoma. Am J Gastroenterol 2013; 108: 200-7.

5. Kastelein F, Spaander MC, Steyerberg EW, et al. Proton pump inhibitors reduce the risk of neoplastic progression in patients with Barrett's esophagus. Clinical gastroenterology and hepatology : the official clinical practice journal of the American Gastroenterological Association 2013; 11: 382-8.

6. Mohamed AA, Mahran KM, Zaazou MM. Impact of laparoscopic Nissen fundoplication on non-complicated Barrett's esophagus. Saudi journal of gastroenterology : official journal of the Saudi Gastroenterology Association 2011; 17: 185-8. 
7. Asari R, Riegler M, Schoppmann SF. Extending Barrett's esophagus cancer risk profile towards genetic abnormalities. Molecular cytogenetics 2013; 6: 10.

8. Benaglia T, Sharples LD, Fitzgerald RC, Lyratzopoulos G. Health benefits and cost effectiveness of endoscopic and nonendoscopic cytosponge screening for Barrett's esophagus. Gastroenterology 2013; 144: 62-73 e6.

9. Grant KS, Demeester SR, Kreger V, et al. Effect of Barrett's esophagus surveillance on esophageal preservation, tumor stage, and survival with esophageal adenocarcinoma. J Thorac Cardiovasc Surg 2013.

10. Mulligan CR, Jr. Multidisciplinary management of esophageal cancer. Surgical oncology clinics of North America 2013; 22: 217-46.

11. Leggett CL, Gorospe EC, Wang KK. Endoscopic therapy for Barrett's esophagus and early esophageal adenocarcinoma. Gastroenterology clinics of North America 2013; 42: 17585.

12. Evans JA, Early DS, Fukami N, et al. The role of endoscopy in Barrett's esophagus and other premalignant conditions of the esophagus. Gastrointest Endosc 2012; 76: 1087-94.

13. 13. Ell C, May A, Pech O, et al. Curative endoscopic resection of early esophageal adenocarcinomas (Barrett's cancer). Gastrointest Endosc 2007; 65: 3-10.

14. May A, Gossner L, Pech O, et al. Local endoscopic therapy for intraepithelial high-grade neoplasia and early adenocarcinoma in Barrett's oesophagus: acute-phase and intermediate results of a new treatment approach. Eur J Gastroenterol Hepatol 2002; 14: 1085-91.

15. Peters FP, Kara MA, Rosmolen WD, et al. Endoscopic treatment of high-grade dysplasia and early stage cancer in Barrett's esophagus. Gastrointest Endosc 2005; 61: 506-14.

16. Guarner-Argente C, Buoncristiano T, Furth EE, Falk GW, Ginsberg GG. Long-term outcomes of patients with Barrett's esophagus and high-grade dysplasia or early cancer treated with endoluminal therapies with intention to complete eradication. Gastrointest Endosc 2013; 77: 190-9.

17. Badreddine RJ, Prasad GA, Wang KK, et al. Prevalence and predictors of recurrent neoplasia after ablation of Barrett's esophagus. Gastrointestinal endoscopy 2010; 71: 697-703.

18. Pech O, Behrens A, May A, et al. Long-term results and risk factor analysis for recurrence after curative endoscopic therapy in 349 patients with high-grade intraepithelial neoplasia and mucosal adenocarcinoma in Barrett's oesophagus. Gut 2008; 57: 1200-6.

19. Pouw RE, Sharma VK, Bergman JJ, Fleischer DE. Radiofrequency ablation for total Barrett's eradication: a description of the endoscopic technique, its clinical results and future prospects. Endoscopy 2008; 40: 1033-40.

20. Castaño R, Rojas A. Evaluación de la eficacia de la terapia ablativa con argón plasma en el esófago de Barrett con displasia: seguimiento prospectivo no menor a un año. Rev Col Gastroenterol 2013; En prensa.

21. Bhat YM, Furth EE, Brensinger CM, Ginsberg GG. Endoscopic Resection with Ligation Using a Multi-Band Mucosectomy System in Barrett's Esophagus with High-
Grade Dysplasia and Intramucosal Carcinoma. Therap Adv Gastroenterol 2009; 2: 323-30.

22. Diaz-Cervantes E, De-la-Torre-Bravo A, Spechler SJ, et al. Banding without resection (endoscopic mucosal ligation) as a novel approach for the ablation of short-segment Barrett's epithelium: results of a pilot study. The American journal of gastroenterology 2007; 102: 1640-5.

23. Fleischer DE, Wang GQ Dawsey $S$, et al. Tissue band ligation followed by snare resection (band and snare): a new technique for tissue acquisition in the esophagus. Gastrointest Endosc 1996; 44: 68-72.

24. Tomizawa Y, Iyer PG, Wong Kee Song LM, Buttar NS, Lutzke LS, Wang KK. Safety of endoscopic mucosal resection for Barrett's esophagus. Am J Gastroenterol 2013; 108: 1440-7; quiz 8.

25. May A, Gossner L, Behrens A, et al. A prospective randomized trial of two different endoscopic resection techniques for early stage cancer of the esophagus. Gastrointest Endosc 2003; 58: 167-75.

26. Schlemper RJ, Riddell RH, Kato Y, et al. The Vienna classification of gastrointestinal epithelial neoplasia. Gut 2000; 47: 251-5.

27. Dixon MF. Gastrointestinal epithelial neoplasia: Vienna revisited. Gut 2002; 51: 130-1.

28. Coupland VH, Lagergren J, Luchtenborg M, et al. Hospital volume, proportion resected and mortality from oesophageal and gastric cancer: a population-based study in England, 2004-2008. Gut 2013; 62: 961-6.

29. Mork H, Barth T, Kreipe HH, et al. Reconstitution of squamous epithelium in Barrett's oesophagus with endoscopic argon plasma coagulation: a prospective study. Scand J Gastroenterol 1998; 33: 1130-4.

30. Byrne JP, Armstrong GR, Attwood SE. Restoration of the normal squamous lining in Barrett's esophagus by argon beam plasma coagulation. Am J Gastroenterol 1998; 93: 1810-5.

31. Manner H, May A, Faerber M, Rabenstein T, Ell C. Safety and efficacy of a new high power argon plasma coagulation system (hp-APC) in lesions of the upper gastrointestinal tract. Digestive and liver disease : official journal of the Italian Society of Gastroenterology and the Italian Association for the Study of the Liver 2006; 38: 471-8.

32. Vargo JJ. Clinical applications of the argon plasma coagulator. Gastrointest Endosc 2004; 59: 81-8.

33. Inoue $\mathrm{H}$, Takeshita $\mathrm{K}$, Hori $\mathrm{H}$, Muraoka $\mathrm{Y}$, Yoneshima $\mathrm{H}$, Endo M. Endoscopic mucosal resection with a cap-fitted panendoscope for esophagus, stomach, and colon mucosal lesions. Gastrointest Endosc 1993; 39: 58-62.

34. Hage M, Siersema PD, van Dekken H, et al. 5-aminolevulinic acid photodynamic therapy versus argon plasma coagulation for ablation of Barrett's oesophagus: a randomised trial. Gut 2004; 53: 785-90.

35. Ragunath K, Krasner N, Raman VS, Haqqani MT, Phillips CJ, Cheung I. Endoscopic ablation of dysplastic Barrett's oesophagus comparing argon plasma coagulation and photodynamic therapy: a randomized prospective trial assessing 
efficacy and cost-effectiveness. Scand J Gastroenterol 2005; 40: 750-8.

36. Kelty CJ, Ackroyd R, Brown NJ, Stephenson TJ, Stoddard CJ, Reed MW. Endoscopic ablation of Barrett's oesophagus: a randomized-controlled trial of photodynamic therapy vs. argon plasma coagulation. Aliment Pharmacol Ther 2004; 20: 1289-96.

37. Ackroyd R, Brown NJ, Stephenson TJ, Stoddard CJ, Reed MW. Ablation treatment for Barrett oesophagus: what depth of tissue destruction is needed? Journal of clinical pathology 1999; 52: 509-12.

38. Barr H, Shepherd NA, Dix A, Roberts DJ, Tan WC, Krasner $\mathrm{N}$. Eradication of high-grade dysplasia in columnar-lined (Barrett's) oesophagus by photodynamic therapy with endogenously generated protoporphyrin IX. Lancet 1996; 348: 584-5.

39. Van Laethem JL, Peny MO, Salmon I, Cremer M, Deviere $\mathrm{J}$. Intramucosal adenocarcinoma arising under squamous re-epithelialisation of Barrett's oesophagus. Gut 2000; 46: 574-7.

40. Bonavina L, Ceriani C, Carazzone A, Segalin A, Ferrero S, Peracchia A. Endoscopic laser ablation of nondysplastic Barrett's epithelium: is it worthwhile? Journal of gastrointestinal surgery : official journal of the Society for Surgery of the Alimentary Tract 1999; 3: 194-9.

41. Shand A, Dallal H, Palmer K, Ghosh S, MacIntyre M. Adenocarcinoma arising in columnar lined oesophagus following treatment with argon plasma coagulation. Gut 2001; 48: 580-1.

42. Gupta M, Iyer PG, Lutzke L, et al. Recurrence of esophageal intestinal metaplasia after endoscopic mucosal resection and radiofrequency ablation of Barrett's esophagus: results from a US Multicenter Consortium. Gastroenterology 2013; 145: 79-86 e1.

43. Fernandez-Sordo JO, Konda VJ, Chennat J, et al. Is Endoscopic Ultrasound (EUS) necessary in the pre-therapeutic assessment of Barrett's esophagus with early neoplasia? Journal of gastrointestinal oncology 2012; 3: 314-21.

44. Bulsiewicz WJ, Dellon ES, Rogers AJ, et al. The impact of endoscopic ultrasound findings on clinical decision making in Barrett's esophagus with high-grade dysplasia or early esophageal adenocarcinoma. Dis Esophagus 2012.

45. Van Laethem JL, Cremer M, Peny MO, Delhaye M, Deviere J. Eradication of Barrett's mucosa with argon plasma coagulation and acid suppression: immediate and mid term results. Gut 1998; 43: 747-51.
46. Pereira-Lima JC, Busnello JV, Saul C, et al. High power setting argon plasma coagulation for the eradication of Barrett's esophagus. Am J Gastroenterol 2000; 95: 1661-8.

47. Schulz H, Miehlke S, Antos D, et al. Ablation of Barrett's epithelium by endoscopic argon plasma coagulation in combination with high-dose omeprazole. Gastrointest Endosc 2000; 51: 659-63.

48. Tigges H, Fuchs KH, Maroske J, et al. Combination of endoscopic argon plasma coagulation and antireflux surgery for treatment of Barrett's esophagus. J Gastrointest Surg 2001; 5: 251-9.

49. Morris CD, Byrne JP, Armstrong GR, Attwood SE. Prevention of the neoplastic progression of Barrett's oesophagus by endoscopic argon beam plasma ablation. $\mathrm{Br} \mathrm{J}$ Surg 2001; 88: 1357-62.

50. Basu KK, Pick B, Bale R, West KP, de Caestecker JS. Efficacy and one year follow up of argon plasma coagulation therapy for ablation of Barrett's oesophagus: factors determining persistence and recurrence of Barrett's epithelium. Gut 2002; 51: 776-80.

51. Kahaleh M, Van Laethem JL, Nagy N, Cremer M, Deviere J. Long-term follow-up and factors predictive of recurrence in Barrett's esophagus treated by argon plasma coagulation and acid suppression. Endoscopy 2002;34:950-5.

52. Garewal H, Ramsey L, Sharma P, Kraus K, Sampliner R, Fass R. Biomarker studies in reversed Barrett's esophagus. Am J Gastroenterol 1999; 94: 2829-33.

53. Krishnadath KK, Wang KK, Taniguchi K, et al. Persistent genetic abnormalities in Barrett's esophagus after photodynamic therapy. Gastroenterology 2000; 119: 624-30.

54. van Hillegersberg R, Haringsma J, ten Kate FJ, Tytgat GN, van Lanschot JJ. Invasive carcinoma after endoscopic ablative therapy for high-grade dysplasia in Barrett's oesophagus. Digestive surgery 2003; 20: 440-4.

55. Manner H, Rabenstein T, Pech O, et al. Ablation of residual Barrett's epithelium after endoscopic resection: a randomized long-term follow-up study of argon plasma coagulation vs. surveillance (APE study). Endoscopy 2014; 46: 6-12.

56. Pouw RE, Bergman JJ. Argon plasma coagulation for Barrett's neoplasia: the right hot ingredient for a successful recipe? Endoscopy 2014; 46: 13-5.

57. Sie C, Bright T, Schoeman M, et al. Argon plasma coagulation ablation versus endoscopic surveillance of Barrett's esophagus: late outcomes from two randomized trials. Endoscopy 2013. 